\title{
Preventing heart failure after STEMI with a cardiac matrix
}

Intracoronary delivery of an injectable bioabsorbable alginate, known as the bioabsorbable cardiac matrix (BCM), does not decrease adverse left ventricular (LV) remodelling or cardiac events at 6 months in patients who underwent stent placement after ST-segment elevation myocardial infarction (STEMI). These findings from the PRESERVATION I trial were published in JACC.

Preclinical studies using murine and swine models of STEMI have shown that BCM could replace damaged extracellular matrix in the infarcted heart to improve LV function and prevent pathological remodelling. The PRESERVATION I trial was performed to assess the safety and efficacy of intracoronary deployment of BCM in patients who underwent percutaneous coronary intervention (PCI) for STEMI.

In this multicentre, randomized, controlled, double-blind trial, 303 patients with STEMI who had a large myocardial infarction despite successful PCI were randomly assigned 2:1 to $\mathrm{BCM}$ or saline injection $(4 \mathrm{ml})$ into the infarct-related artery 2-5 days after primary PCI. BCM is an aqueous solution made up of $1.0 \%$ sodium alginate and $0.3 \%$ calcium gluconate. The primary efficacy end point was change in LV end-diastolic volume index (LVEDVI) measured on transthoracic echocardiography from time of BCM deployment to the 6-month follow-up. Secondary efficacy end points included functional and quality-of-life tests, NYHA functional class, and 6-min walking test. The primary safety end point was a composite of cardiovascular death, recurrent myocardial infarction, target-vessel revascularization, stent thrombosis, substantial arrhythmia necessitating surgery, or myocardial rupture through 6 months.

In total, 201 patients received $\mathrm{BCM}$ and 102 received saline control. No significant difference was observed $\stackrel{5}{>}$ in LVEDVI between the
two treatment groups at the 6-month follow-up (mean follow-up (mean
change from baseline to 6 months $\pm \mathrm{SD}$ : $\mathrm{BCM} 14.1 \pm 28.9 \mathrm{ml} / \mathrm{m}^{2}$ versus saline $11.7 \pm 26.9 \mathrm{ml} / \mathrm{m}^{2}$ $P=0.49)$. Furthermore, no differences between treatment groups were detected for secondary efficacy end points, but a nonsignificant trend towards improved 6-min walking test was observed in the BCM group $(P=0.051)$. Primary safety end points were similar between the BCM and the saline control groups, as was the incidence of adverse events (76.1\% versus $74.5 \%$ ).

Although these results do not support earlier positive findings in preclinical models, the researchers are considering alternative methods of delivery for future studies. According to Sunil Rao, lead investigator of the study, these include "administering the BCM at a different time point in the treatment of STEMI, or using $\mathrm{BCM}$ as a vehicle for cells that might regenerate the heart muscle".

In an accompanying editorial, Antoni Bayés-Genís, Carolina Gálvez-Montón, and Santiago Roura questioned whether the chosen deployment volume $(4 \mathrm{ml})$, use of LVEDVI as the primary end point, and the delivery route were appropriate for this study, and emphasized that "further insights into the mechanisms, route of delivery, timing, and clinical setting are mandatory to minimize translational failure".

Karina Huynh

ORIGINAL ARTICLE Rao, S. V.et al.

Bioabsorbable intracoronary matrix for prevention

of ventricular remodeling after myocardial

infarction. J. Am. Coll. Cardiol. 68, 715-723 (2016) 